This material is posted here with permission of the IEEE. Such permission of the IEEE does not in any way imply IEEE endorsement of any of Helsinki University of Technology's products or services. Internal or personal use of this material is permitted. However, permission to reprint/republish this material for advertising or promotional purposes or for creating new collective works for resale or redistribution must be obtained from the IEEE by writing to pubs-permissions@ieee.org.

By choosing to view this document, you agree to all provisions of the copyright laws protecting it. 


\title{
Design of a Temperature-Stable RF MEM Capacitor
}

\author{
Heikki Nieminen, Vladimir Ermolov, Samuli Silanto, Kjell Nybergh, and Tapani Ryhänen
}

\begin{abstract}
This paper presents a novel temperature-compensated two-state microelectromechanical (MEM) capacitor. The principle to minimize temperature dependence is based on geometrical compensation and can be extended to other devices such as MEM varactors. The compensation structure eliminates the effect of intrinsic and thermal stress on device operation. This leads to a temperature-stable device without compromising the quality factor $(Q)$ or the voltage behavior. The compensation structure increases the robustness of the devices, but does not require any modifications to the process. Measurement results verify that the OFF and ON capacitance change is less than $6 \%$ and the pull-in voltage is less than $5 \%$ when the temperature is varied from -30 to $+70{ }^{\circ} \mathrm{C}$.

[1066]
\end{abstract}

Index Terms-Microelectromechanical (MEM), RF MEMS, temperature compensation.

\section{INTRODUCTION}

$\mathbf{T}$ HERE is a steady tendency in wireless technology toward systems that can be reconfigured and fine-tuned to achieve optimal performance. In addition, the components in these RF systems require increasingly higher $\mathrm{Q}$. There are many approaches to these challenges depending on the particular system level requirements. One solution is MEM capacitor technology.

The added values a MEM capacitor brings to the RF system are high linearity, high-Q, large capacitance variation, ability to handle high RF power, and hot switching ability [1]-[4]. The most significant drawbacks of the technology include large temperature dependence, high control voltage, slow speed, and size and cost of the component. The high control voltage is probably the biggest drawback when considering the possible system applications. A dc/dc converter must be integrated with the control electronics. This increases the overall cost, size, and power consumption. However, in some applications the advantages of MEM capacitors can exceed the drawbacks.

During recent years, great progress has been made in MEM capacitor development. To increase the $\mathrm{Q}$ of the device, metal has been selected as the structural material [2]-[5]. The development of the packaging has led to wafer-level hermetic packaging solutions that decrease the size and the price of the component [6]-[8]. In addition, the research of electrical reliability has provided a path toward reliable components [9]. However, one challenge remaining with the parallel-plate metal MEM capacitor is temperature stability without compromising the capacitance as a function of the voltage behavior, speed, and the $\mathrm{Q}$ of the device.

Manuscript received May 28, 2003; revised January 8, 2004. Subject Editor N. C. Tien.

The authors are with the Nokia Research Center, FIN-00045 Nokia Group, Finland (e-mail: heikki.nieminen@ nokia.com).

Digital Object Identifier 10.1109/JMEMS.2004.832192

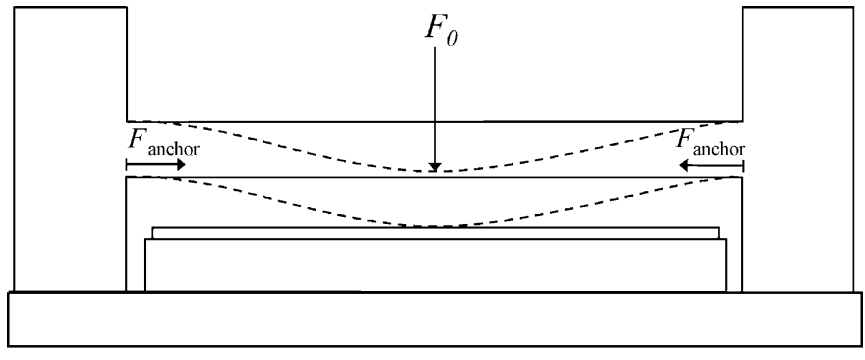

Fig. 1. A simple bridge capacitor is depicted. $F_{\text {anchor }}$ represents the forces that the fixed anchoring generates on the suspended electrode, when the electrode expands or contracts due to temperature change. $F_{0}$ is the perturbation force, for instance, the electrostatic force due to the control voltage.

Section II of this paper explains why MEM capacitor has temperature dependence. Then possible solutions to minimize temperature dependence are presented. Section III describes in detail the design of the temperature-compensated MEM capacitor. Measurement results of the fabricated components are in Section IV. Finally, conclusions are presented in Section V.

\section{TEMPERATURE DEPENDENCE OF MEM CAPACITOR}

The temperature dependence of the metal bridge capacitor is due to the thermal expansion and contraction mismatch between the suspended electrode and the substrate. The thermal expansion and contraction of the suspended electrode is restricted by the fixed anchors. This causes a force $\left(F_{\text {anchor }}\right)$ to act on the suspended electrode, as shown in Fig. 1. Consequently, this force causes a stress on the suspended electrode. We will refer to this as thermal stress. Depending on the temperature, thermal stress can be either tensile or compressive. In addition to the thermal stress, the suspended bridge also has some postfabrication residual stress.

The residual stress and the thermal stress are summed to get the total stress in the suspended electrode at different temperatures. The total stress in the membrane strongly affects the capacitance as a function of the voltage behavior of the device. The increase in tensile stress of the bridge strongly increases the pull-in voltage. In addition, when the tensile stress in the bridge reaches high enough level, the metal yields.

The compressive stress causes more dramatic changes in the capacitance as a function of the voltage behavior. When compressive stress increases, a buckling occurs due to the slightest perturbation force $F_{0}$. The control voltage is a strong perturbation force. Therefore, the capacitance as a function of the voltage behavior can be expected to change due to the compressive stress. In the worst case, buckling occurs and the suspended electrode remains in contact with the lower electrode even after the control voltage is reduced to zero. 


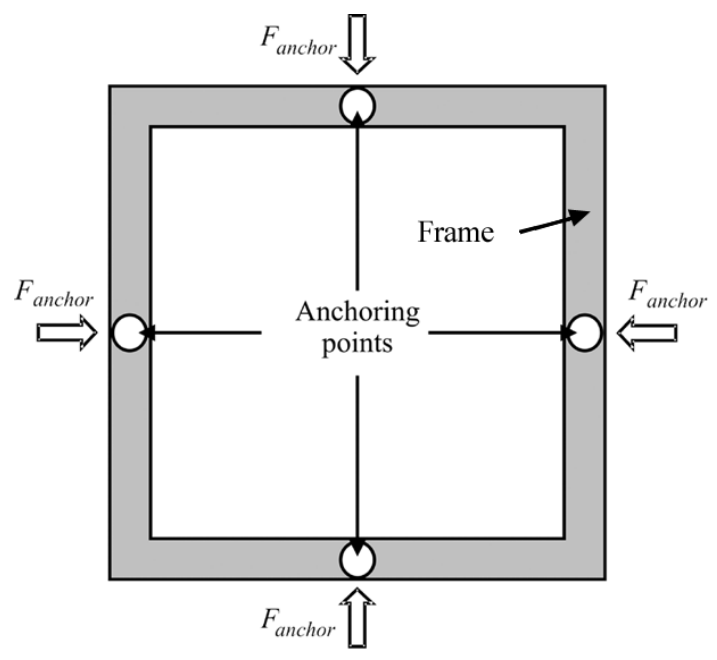

(a)

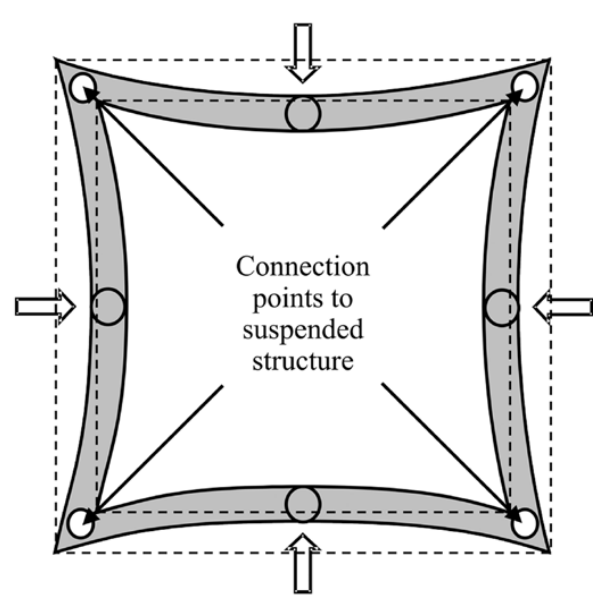

(b)

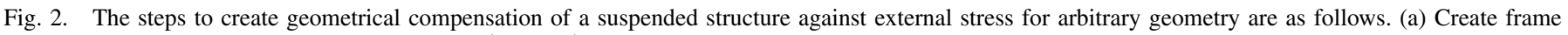

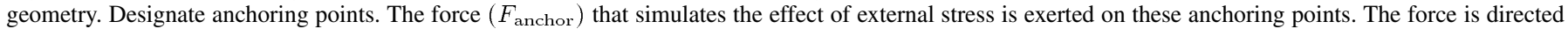

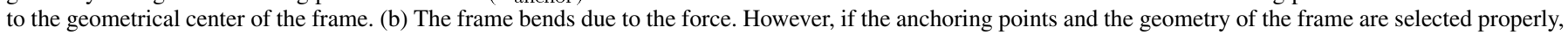
there are points in the frame that do not move or move very slightly. These are points where the frame is connected to the suspended structure.

There are several ways to avoid harm to the device operation that is caused by thermal stress. The first way is to connect the suspended electrode to the substrate only from one point [1], [10]-[12]. Consequently, it can freely expand and contract due to temperature change. However, the resulting structure warps easily due to the intrinsic stress gradient. This decreases the repeatability of the manufacturing process. In addition, the capacitor as a function of voltage performance is not optimal since the device will be asymmetric. The second way to decrease the thermal stress is to use springs [2], [3], [13]. The springs are placed between the anchor points and the suspended structure so that they reduce the thermal stress anchors induced into the membrane. This method has serious drawback that the capacitance as a function of voltage behavior of the device is affected by the temperature compensation structure.

The third way to decrease thermal stress in the suspended structure is to design thermal compensation structure that eliminates force created by thermal stress in certain points [14]. When suspended structure is connected to these points no thermal stress is exerted into it. We will refer to this as geometrical compensation of thermal stress. The advantage of the geometrical compensation is that the temperature compensation does not interfere with the voltage behavior of the device.

\section{DESIGN OF MEM CAPACITOR}

\section{A. Capacitance as a Function of Voltage Behavior}

Two-state MEM capacitor design starts from the definition of the OFF $\left(C_{\mathrm{OFF}}\right)$ and $\mathrm{ON}$ capacitance $\left(C_{\mathrm{ON}}\right)$ ratio. This in turn defines the pull-in $\left(U_{\text {pull-in }}\right)$ and release voltage $\left(U_{\text {release }}\right)$ ratio. $U_{\text {release }}$ is defined as the smallest voltage that keeps the capacitor in the ON state. It is known that [15]

$$
U_{\text {pull-in }}^{2}=\left(\frac{2}{3}\right)^{3} \frac{\varepsilon_{0}^{2} A^{2} k_{\text {spring }}}{C_{\mathrm{OFF}}^{3}}
$$

and

$$
U_{\text {release }}^{2}=\frac{2 k_{\text {spring }} \varepsilon_{0} A t_{\text {gap }}}{C_{\mathrm{ON}}^{2}}
$$

where $t_{\text {gap }}$ is the air gap, A is the area of the electrode, $\varepsilon_{r}$ is the dielectric constant, and $k_{\text {spring }}$ is the spring constant. The ratio between $U_{\text {release }}$ and $U_{\text {pull-in }}$ follows from (1) and (2)

$$
\frac{U_{\text {release }}^{2}}{U_{\text {pull-in }}^{2}}=\frac{C_{\mathrm{OFF}}^{2}}{C_{\mathrm{ON}}^{2}}\left(\frac{3}{2}\right)^{3}\left(\frac{2 \varepsilon_{r} t_{\text {gap }}}{t_{\text {dielectric }}+\varepsilon_{r} t_{\text {gap }}}\right)
$$

where $t_{\text {dielectric }}$ is the dielectric layer thickness. If the ON and OFF capacitance ratio is defined as

$$
\alpha=\frac{C_{\mathrm{OFF}}}{C_{\mathrm{ON}}}
$$

(3) becomes

$$
\frac{U_{\text {release }}}{U_{\text {pull-in }}}=\alpha\left(\frac{3}{2}\right)^{\frac{3}{2}} \sqrt{2(1-\alpha)} .
$$

Since the capacitance ratio in practice defines $t_{\text {dielectric }}$ and $t_{\text {gap }}$, the design parameter that is free for control voltage tuning is $k_{\text {spring. }}$. Here, the spring force is assumed to be linear. It must be kept in mind that whenever large deflection occurs, this assumption is no longer valid. As a result, estimation for the pull-in voltage (1) is more accurate than estimation for the release voltage (2).

Assuming that the RF voltage signal is sinusoidal $\left(\hat{u}_{\mathrm{RF}} \sin \omega t\right)$, it generates a force [3] that is equivalent to that caused by a dc voltage $\left(U_{\mathrm{RF}}\right)$ over the capacitor according to

$$
U_{\mathrm{RF}}=\frac{\hat{u}_{\mathrm{RF}}}{\sqrt{2}}
$$



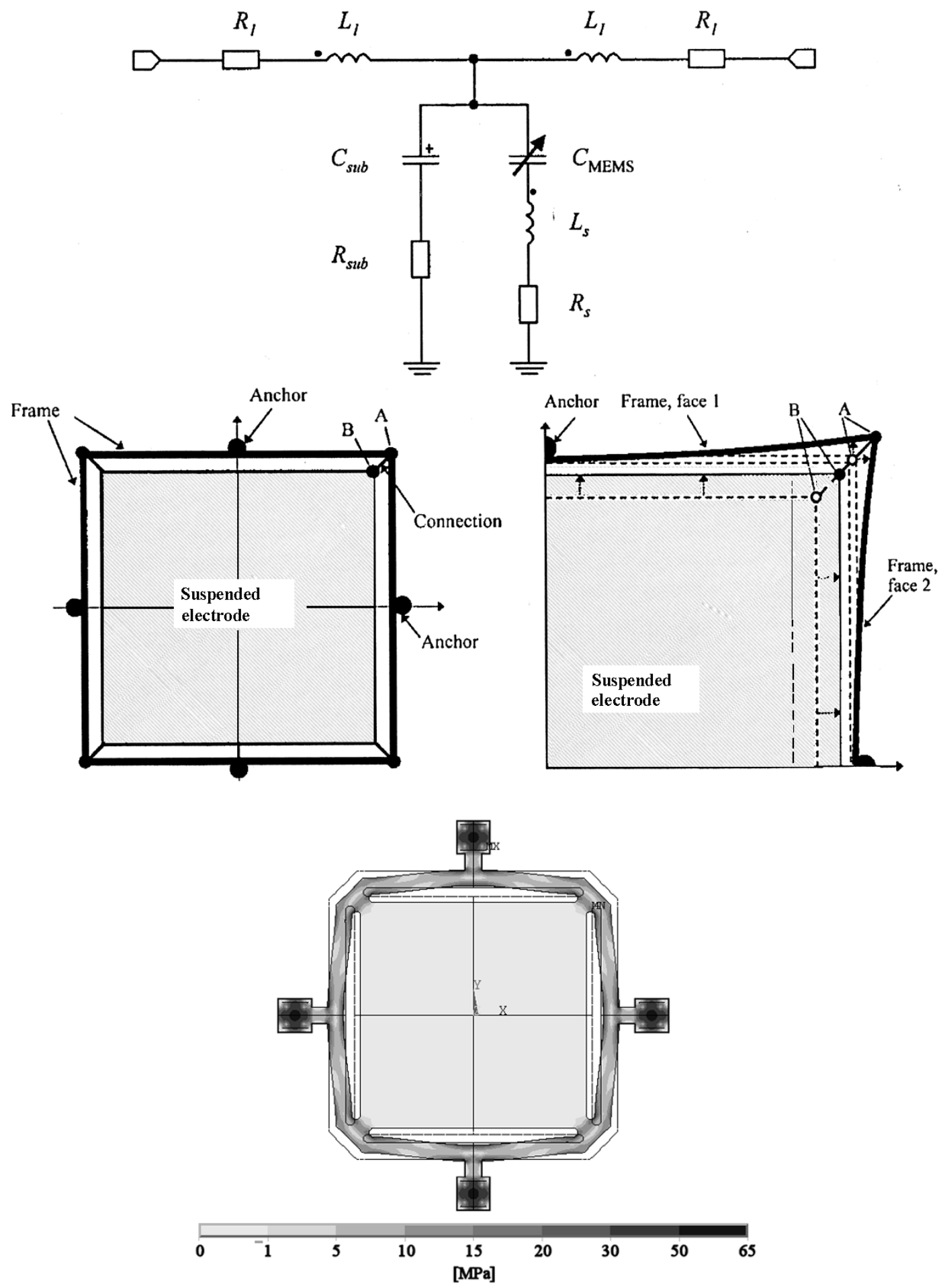

Fig. 3. FEM analysis of the temperature-compensated capacitor. Simulation is done with ANSYS. The temperature change is $-50{ }^{\circ} \mathrm{C}$. The substrate is silicon. The suspended electrode, the frame and the anchors are $4-\mu \mathrm{m}$-thick gold. The scale at the bottom of the figure shows stress. Displacements in the figure are exaggerated.

This is one of the design constraints for $U_{\text {release. The }} U_{\text {release }}$ voltage of the device must be larger than the $U_{\mathrm{RF}}$ generated by the RF voltage signal. Only in that case can the MEM capacitor go from the ON state to the OFF state when the control voltage is switched to zero but the RF voltage signal is still over the device. However, ON-to-OFF-state switching speed $\left(\tau_{\mathrm{OFF}}\right)$ sets an even higher limit for the release voltage. The RF voltage signal over the MEM capacitor causes a force that tries to pull the suspended membrane down. Therefore, when the device is switched from the ON state to the OFF state, the RF voltage signal increases $\tau_{\text {OFF }}$ preventing switching totally when the $U_{\mathrm{RF}}$ reaches $U_{\text {release. }}$ Other important design parameters affecting $\tau_{\mathrm{OFF}}$ are ambient gas pressure $\left(p_{\text {gas }}\right)$, size $\left(d_{\text {hole }}\right)$ and pitch $\left(d_{\text {pitch }}\right)$ of the sacrificial etching holes. Veijola et al. [16] have presented a system level model that can be used to evaluate the effect of these parameters on $\tau_{\mathrm{OFF}}$. 
The pull-in voltage has also design limits. One is the available control voltage. The other limit is the maximum electric field $\left(E_{\max }\right)$ allowed in the dielectric layer before dielectric charging starts to degrade the operation. The latter limit can be partially circumvented using a reduced hold-down voltage $\left(U_{\text {hold }}\right)$ [9]. However, since the capacitance value during contact can vary depending on the control voltage, FEM simulations must be done to ensure that the ON capacitance value remains as desired. As a result, the electrical reliability sets a minimum limit for $t_{\text {dielectric }}$ that is dependent on the $U_{\text {hold }}$. This in turn limits the maximum ON capacitance density.

\section{B. Compensation of Temperature Dependence}

The following outlines the steps to create geometrical compensation of a suspended structure against external stress for arbitrary geometry. Step one, create frame geometry. Step two, designate anchoring points where the frame will be connected to the substrate. This is shown in Fig. 2(a). Step three, exert force $\left(F_{\text {anchor }}\right)$ that simulates effect of external stress on these anchoring points. The force is directed to the geometrical center of the frame. Step four, simulate or calculate how the frame bends due to the force. If the anchoring points and the geometry of the frame are selected properly, there are points in the frame that do not move or rotate. If the point of the frame is not moving or rotating due to the external force, the net force and torque acting on this point is zero. Therefore, if the suspended structure is connected to the frame from this point, no force (due to external stress) is exerted to the suspended structure from the frame. This is shown in Fig. 2(b). This design loop is repeated, changing the anchoring points and the shape of the frame until achieving the result that meets the design constraints. Because the operation principle is geometrical, the spring constant of the bending frame is irrelevant. This means that the frame can be made very thick in order to have minimal parasitic resistance and inductance.

A special case of geometrical compensation that is given more consideration is a square frame around a square-shaped suspended membrane. The anchoring points are selected to be at the center of each face of the square. When the force that the external stress exerts into the anchoring points bends the frame, the corners of the frame remain almost motionless. Therefore, the suspended membrane is connected to the frame at the corners. Fig. 3 shows simulated performance of such a frame. A three-dimensional (3-D) finite element method (FEM) simulation, using the ANSYS simulator, shows that a $-50{ }^{\circ} \mathrm{C}$ temperature change causes less than $1 \mathrm{MPa}$ tensile stress into the suspended electrode. In addition, similar simulation with a $+50{ }^{\circ} \mathrm{C}$ temperature change shows that less than $1 \mathrm{MPa}$ compressive stress is exerted into the suspended electrode. The substrate in the simulations is silicon and the suspended electrode, the frame and the anchors are 4- $\mu \mathrm{m}$-thick gold. The other dimensions of the device are shown in Fig. 6 and in Table I.

In addition to improved thermal stability, this simulation also demonstrates that the operation of the device is independent of the in-plane intrinsic stress in the metal structure. In-plane stress will always be released from the suspended membrane. This increases a lot the repeatability of the metal RF MEMS process.

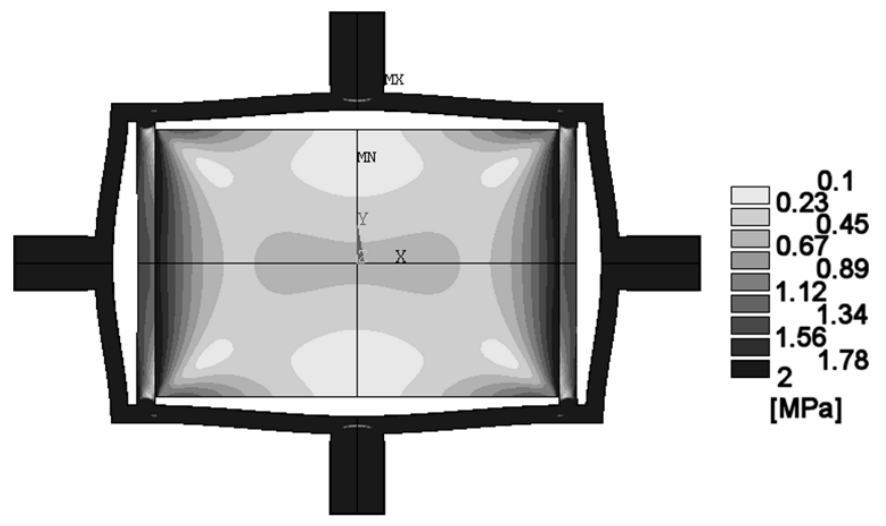

Fig. 4. The FEM simulation of the temperature-compensated bridge capacitor. The frame is $10-\mu \mathrm{m}$-thick and $20-\mu \mathrm{m}$-wide gold and the membrane is $0.5-\mu \mathrm{m}$-thick gold. A temperature change of $-55^{\circ} \mathrm{C}$ causes only less than $2 \mathrm{MPa}$ stress to the suspended electrode. The scale in the right of the figure shows stress. Displacements in the picture are exaggerated.

TABLE I

LAYER THICKNESS

\begin{tabular}{l|r}
\hline Layer & Thickness \\
\hline Metal 1 & $0.8 \mu \mathrm{m}$ \\
\hline Dielectric layer & $0.1 \mu \mathrm{m}$ \\
\hline Sacrificial layer (Polymer) & $1.2 \mu \mathrm{m}$ \\
\hline Metal 2 & $4.0 \mu \mathrm{m}$ \\
\hline
\end{tabular}

On the other hand, the vertical stress gradient still causes challenges. But the effect is not as severe as when the device is anchored only from one point. This is due to the symmetrical placing of anchor points. The air gap at the center of the suspended electrode is the same height as defined by the sacrificial layer. The vertical stress gradient bends only the corner of the suspended electrode up or down depending on the stress gradient. This will affect the voltage behavior. The pull-in voltage will increase or decrease depending on the warping direction because the air gap changes at the corners.

The geometrical compensation principle described here can be applied to any device that requires isolation from the stresses generated in the substrate. One such example is the temperature-compensated bridge capacitor that is shown in Fig. 4. The frame is made of thick metal to prevent its movement perpendicular to the substrate. The suspended electrode, which forms the bridge capacitor, can be made from a thin metal film that is connected from its ends to the frame. Fig. 4 shows the FEM simulation result of the device. The frame is $10-\mu \mathrm{m}$-thick and $20-\mu \mathrm{m}$-wide gold and the suspended electrode is $0.5-\mu \mathrm{m}$-thick gold. A temperature change of $-55^{\circ} \mathrm{C}$ causes less than $2 \mathrm{MPa}$ stress to the suspended electrode.

However, unlike the previous device that is made from a single metal layer, this device is sensitive to the intrinsic in-plane stress of the metal layers. The stress difference between thick metal forming the frame and thin metal forming the suspended electrode remains in the thin metal membrane. This is an advantage since the increase in the tensile stress of the 

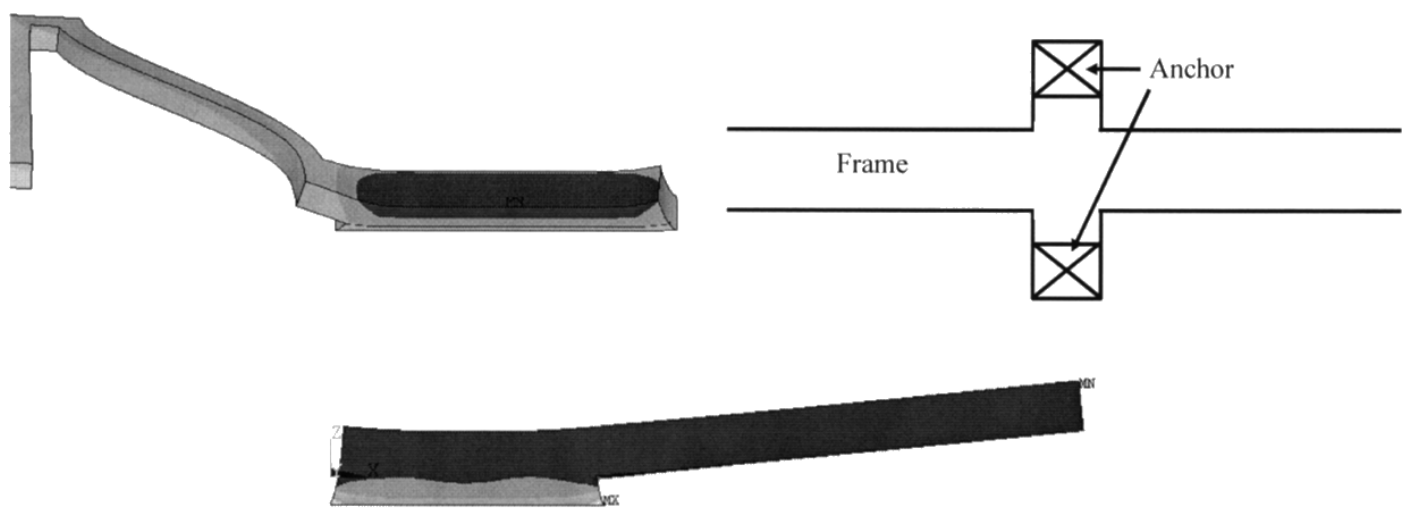

(a)

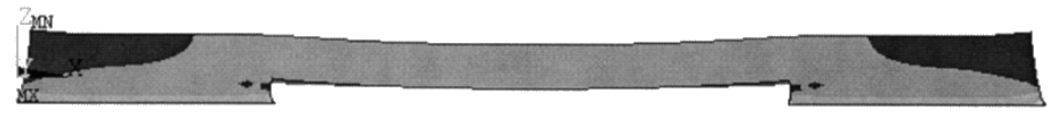

(b)

Fig. 5. (a) Cross-section view of the simple anchor that causes temperature dependence of the MEM capacitor. The simulation was done with ANSYS. (b) Crosssection view of the symmetrical anchoring that minimizes the temperature dependence that the anchors cause to the MEM capacitor. The displacement caused by the thermal contraction is increased 150 times in both (a) and (b).

membrane increases the operation speed of the device. However, if the stress after release in the membrane is compressive, the membrane may buckle.

\section{Anchoring}

Simulations show that the frame prevents most of the thermal stress on the suspended electrode. However, they also show that the capacitance still changes due to the temperature variation caused by the anchors. Fig. 5(a) shows the cross section of the anchor that is simulated by FEM. The top part of the anchor is free to contract due to temperature change. However, the bottom of the anchor is fixed to the substrate. Since the substrate has smaller thermal expansion coefficient than the anchor, the bottom of the anchor contracts less than the upper part of the anchor. This exerts torque to the frame that will displace the suspended electrode. Because the suspended electrode usually has large width and length compared to the air-gap height, the displacement due to torque can be quite considerable.

Torque from anchor can be eliminated, for instance, using the symmetrical anchoring. Cross section of symmetrical anchor that is simulated by FEM is shown in Fig. 5(b). The two anchor points located at opposite sides of the frame cancel each other's torque. The best way to place the two anchor points is perpendicular to the frame. However, this may be difficult due to closeness of the suspended electrode.

\section{Temperature-Compensated Capacitor Design}

Fig. 6 shows a simplified top and cross-section view of the temperature-compensated "Square" capacitor. The dimensions of the device are shown in Fig. 6 and in Table I. Metal 1 and 2 are gold thin films. The design goals for the capacitor were $C_{\mathrm{ON}}=$
$19 \mathrm{pF}, C_{\mathrm{OFF}}=0.65 \mathrm{pF}$, and pull-in voltage $U_{\text {pull-in }}<20 \mathrm{~V}$. The dielectric layer on top of the lower electrode is $100-\mathrm{nm}$ thick silicon nitride. The average air gap remaining between the suspended electrode and the dielectric layer due to surface roughness is assumed to be $15 \mathrm{~nm}$. The releasing process dictates the size of the sacrificial etching holes and their pitch in the suspended electrode. The holes are $10 \times 10 \mu \mathrm{m}^{2}$ squares with a pitch of $20 \mu \mathrm{m}$. Consequently, the size of the suspended electrode must be $280 \mu \mathrm{m} \times 280 \mu \mathrm{m}$. An air gap of $1.2 \mu \mathrm{m}$ is, thus, determined to achieve the required $C_{\mathrm{OFF}}$ value.

The frame defines the capacitance as a function of the voltage of the device. For calculation purposes, the frame can be estimated to be composed of a system that has eight parallel cantilevers. Since the thickness of the suspended metal is a fixed process parameter, the spring constant can be changed by changing the width of the frame. The length of the spring is $96 \mu \mathrm{m}$. This is depicted in Fig. 6. Using (1) gives a pull-in voltage of $13.3 \mathrm{~V}$ for a $20-\mu \mathrm{m}$-wide frame. On the other hand, FEM simulation gives a pull-in voltage of $13.1 \mathrm{~V}$.

\section{E. Electrical Parameters}

Fig. 7 shows the electrical equivalent circuit of the MEM capacitor. In the equivalent circuit, the suspended electrode (metal two) and the substrate are connected to the ground. Metal one forms the signal line that passes under the suspended electrode. $C_{\text {MEMS }}$ is the capacitance between the signal line and the suspended electrode. $C_{\text {sub }}$ is the capacitance between the signal line and substrate. $R_{1}$ and $L_{1}$ are the signal line resistance and inductance, respectively. $R_{\text {sub }}$ is the substrate resistance. $L_{s}$ and $R_{s}$ are the inductance and resistance of the suspended electrode and the frame. 

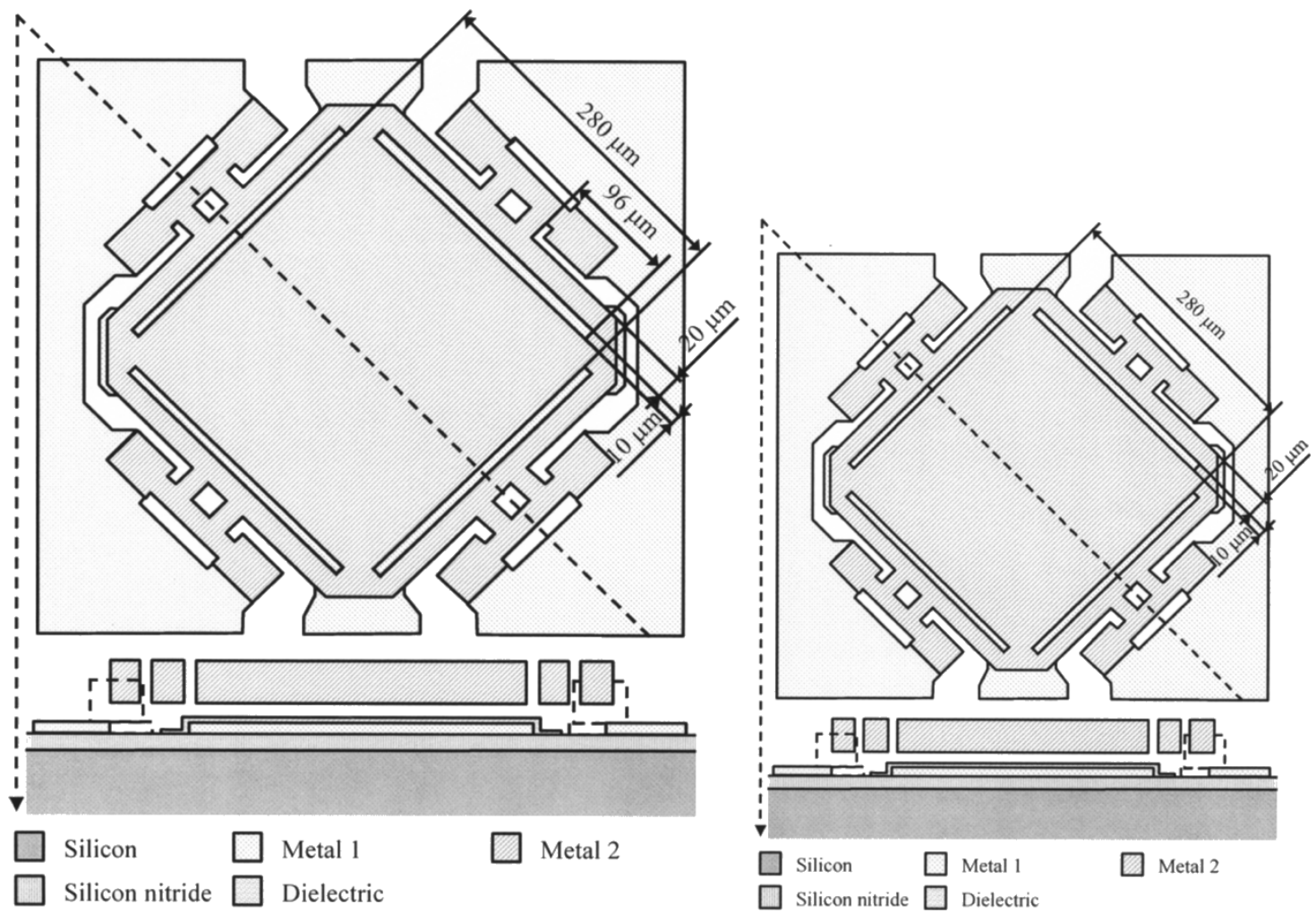

Fig. 6. The top and cross-section view of the square capacitor.

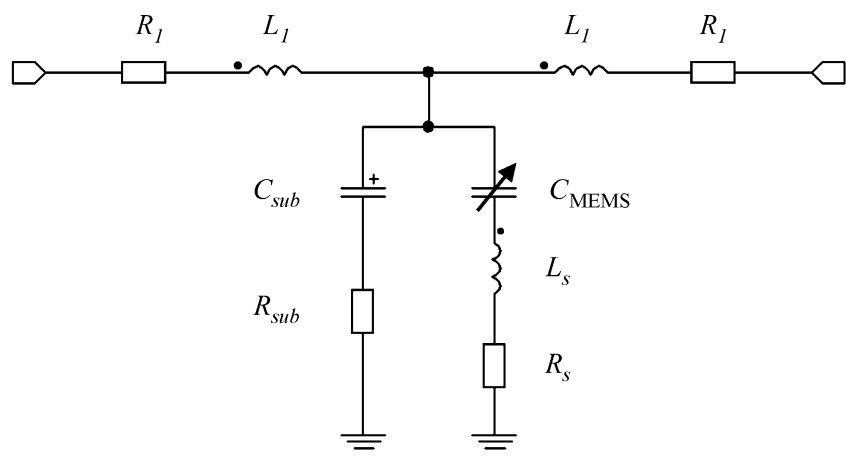

Fig. 7. Electrical equivalent circuit of the MEM capacitor. The suspended electrode (metal two) and the substrate are connected to the ground. $C_{\mathrm{MEMS}}$ is the capacitance between the signal line and the suspended electrode. $C_{\mathrm{sub}}$ is the capacitance between the signal line and substrate. $R_{1}$ and $L_{1}$ are the signal line resistance and inductance, respectively. $R_{\text {sub }}$ is the substrate resistance. $L_{s}$ and $R_{s}$ are the inductance and resistance of the suspended electrode and the frame.

\section{RESULTS AND DISCUSSION}

\section{A. Measurement Theory}

The devices in this paper are designed to operate in the RF frequency range from 0.9 to $2 \mathrm{GHz}$. The substrate material is high-resistivity silicon. At low frequencies, the parasitic substrate capacitance that is parallel to the MEM capacitor increases the measured capacitance value. However, in frequencies from 0.9 to $2 \mathrm{GHz}$ the substrate capacitance increases the measured capacitance only slightly. Therefore, the capacitance is calculated from the measured S-parameters in the frequency range of interest. In this paper two different measurement setups are used to measure the S-parameters. A one-port mea-

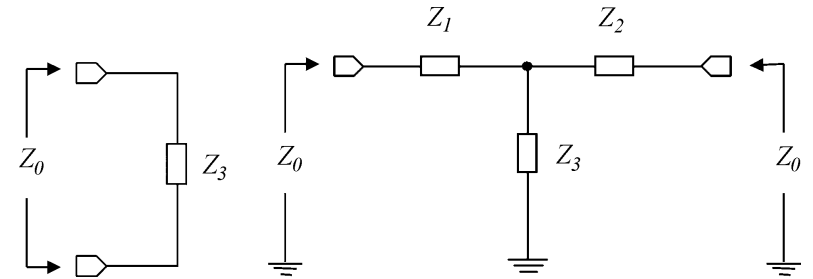

(a)

(b)

Fig. 8. Depicted in (a) is the one port measurement setup used for the transient capacitance measurement. Depicted in (b) is a T-network and its relationship to Z-parameters.

surement setup is used to calculate the transient capacitance and a two-port measurement setup is used to calculate the steady-state capacitance. Fig. 8(a) and (b) shows what kind of network is used to model the measured device in a one- and a two-port measurement setup, respectively. In order to calculate the capacitance $C$, the impedance $Z_{3}$ must be calculated from the measured S-parameters. The impedance from the two-port $\mathrm{S}$-parameters is

$$
Z_{3}=Z_{0} \frac{2 S_{21}}{\left(1-S_{11}\right)\left(1-S_{22}\right)-S_{12} S_{21}}
$$

and the impedance from the one-port S-parameter is

$$
Z_{3}=Z_{0} \frac{1+S_{11}}{1-S_{11}}
$$

The capacitance from the impedance is

$$
C=-\frac{1}{\omega \operatorname{Im}\left[Z_{3}\right]} .
$$




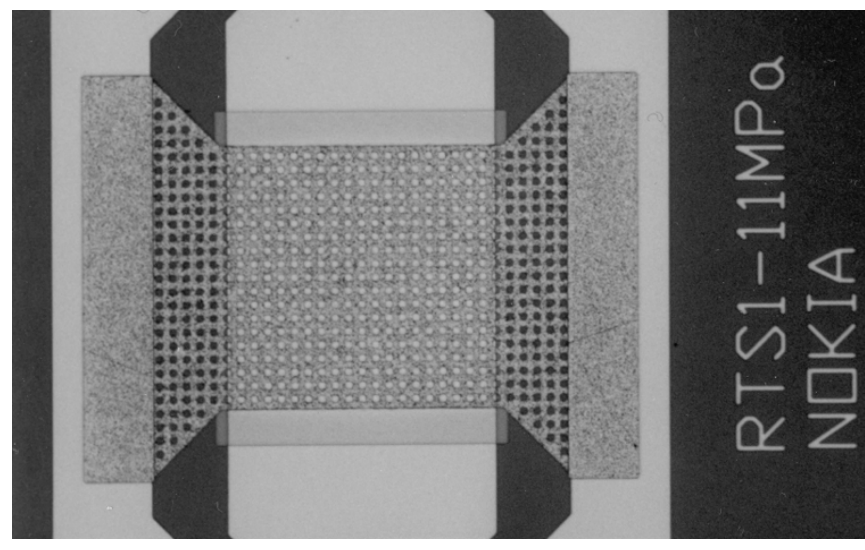

Fig. 9. Photograph of a bridge capacitor.

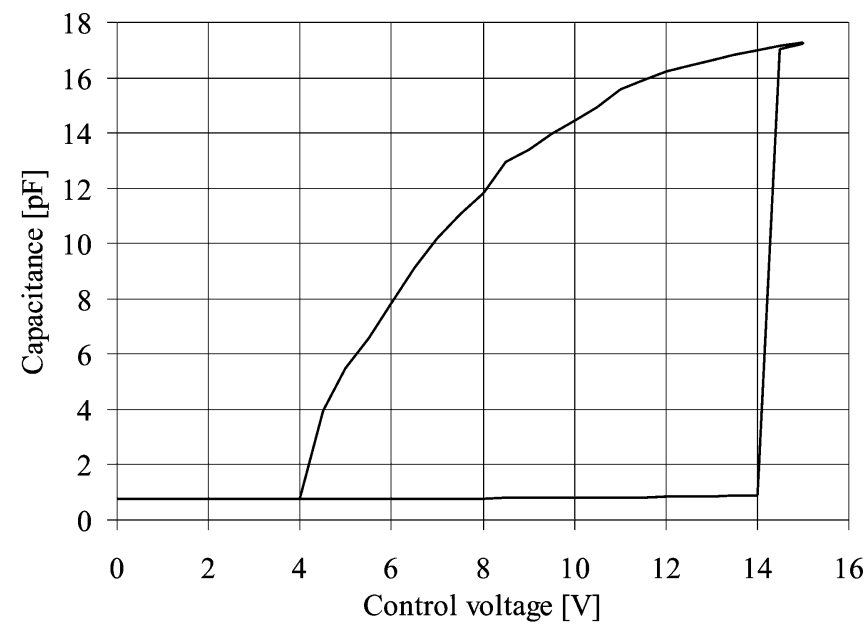

(a)

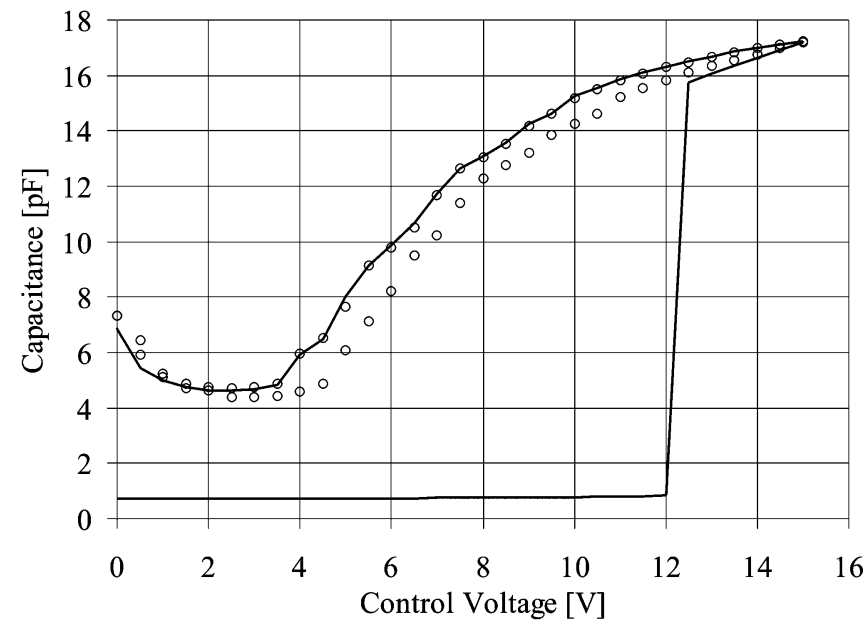

(b)

Fig. 10. Temperature dependence of the bridge capacitor. (a) Is the capacitance as a function of voltage at $+25^{\circ} \mathrm{C}$. (b) Shows the capacitance as a function of voltage at $+55{ }^{\circ} \mathrm{C}$ during the first (continuous line) and the second (circles) control voltage cycle.

\section{B. Measurement Equipment}

All measurements were carried out using a Karl Suss PA 200-II probe station that has temperature control and a dry airflow system. The relative humidity of the air in the measurement chamber was monitored with Vaisala's DM70 Dewpoint Meter.
TABLE II

Equivalent Circuit Values Calculated From MEASURED S-PARAMETERS

\begin{tabular}{l|r}
\hline Parameter & Value \\
\hline$C_{\text {MEMS_off }}$ & $1.0 \mathrm{pF}$ \\
\hline$C_{\text {MEMS_on }}$ & $7.0 \mathrm{pF}$ \\
\hline$R_{s}$ & $25 \mathrm{~m} \Omega$ \\
\hline$L_{s}$ & $15 \mathrm{pH}$ \\
\hline$C_{\text {sub }}$ & $0.27 \mathrm{pF}$ \\
\hline$R_{\text {sub }}$ & $780 \Omega$ \\
\hline$R_{I}$ & $36 \mathrm{~m} \Omega$ \\
\hline$L_{l}$ & $0.1 \mathrm{nH}$ \\
\hline
\end{tabular}
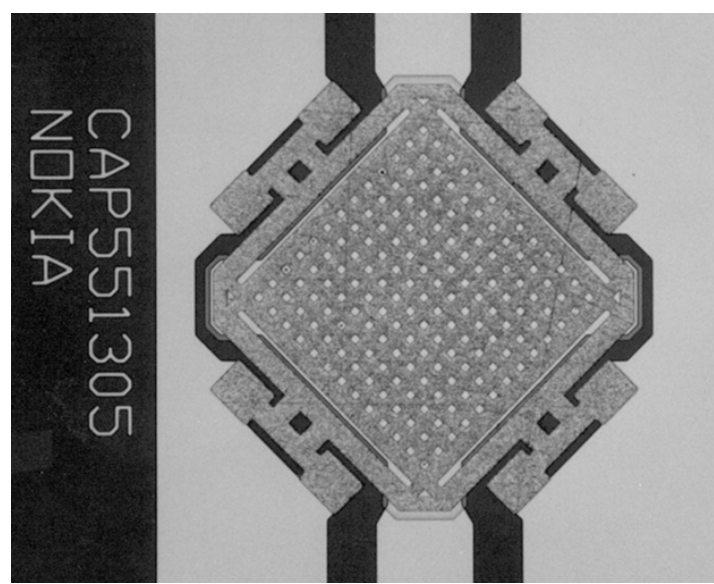

Fig. 11. Photograph of a temperature-compensated capacitor.

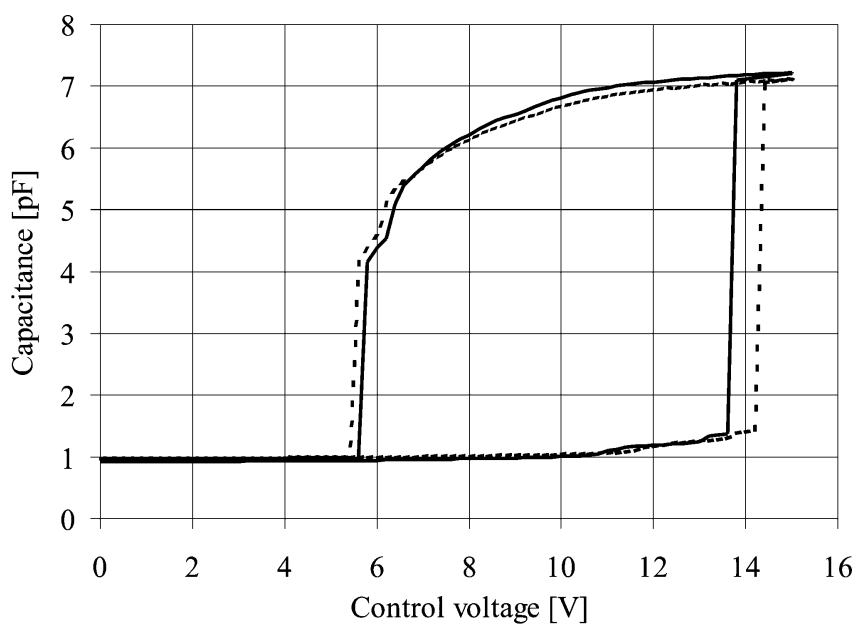

Fig. 12. Measured temperature dependence of the square capacitor. The dashed line is the capacitance as a function of the voltage at $-30^{\circ} \mathrm{C}$ and the continuous line is the capacitance as a function of the voltage at $+70^{\circ} \mathrm{C}$. Over the measured temperature range, the fluctuation of $C_{\mathrm{OFF}}$ is less than $6 \%, C_{\mathrm{ON}}$ less than $2 \%$, and $U_{\text {pull-in }}$ less than $5 \%$.

During the measurements the relative humidity in the chamber was below $0.05 \%$ and the air temperature was $23{ }^{\circ} \mathrm{C}$. The S-parameters as a function of voltage were measured using a Rohde 


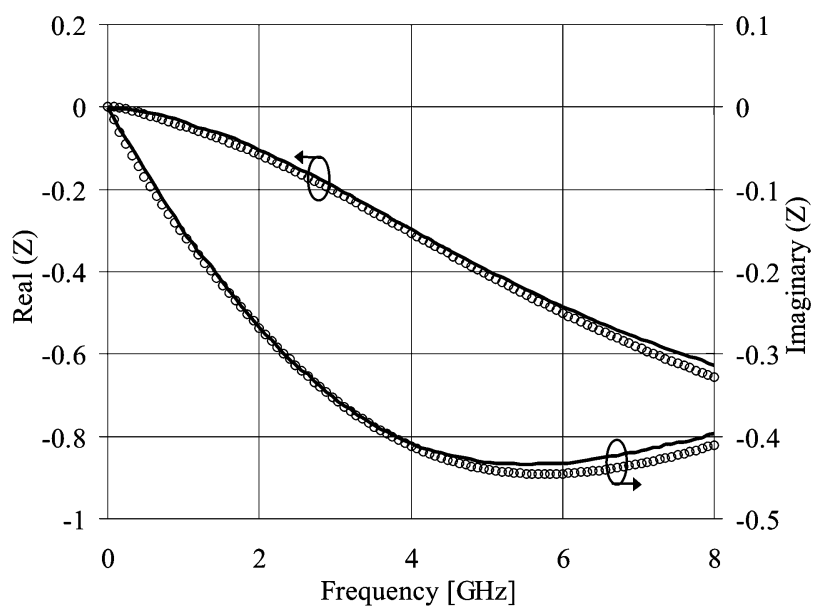

(a)

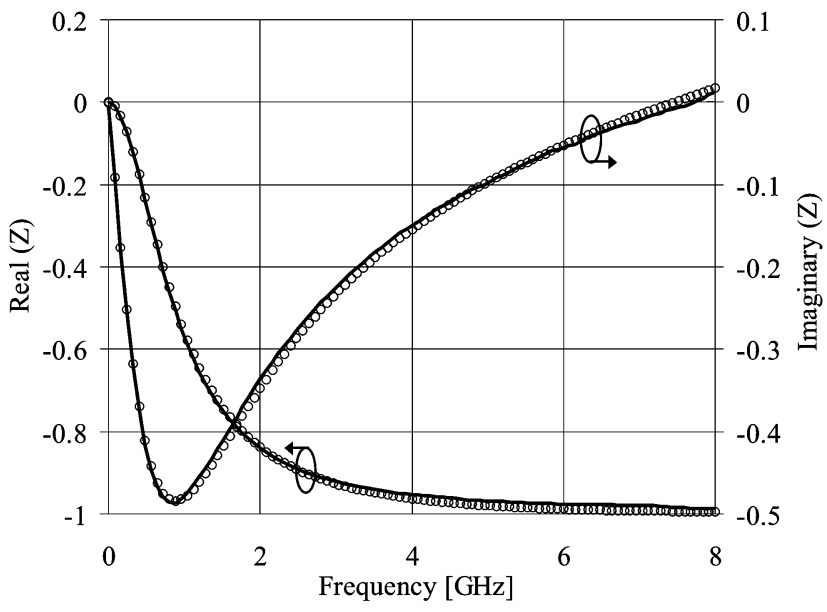

(c)

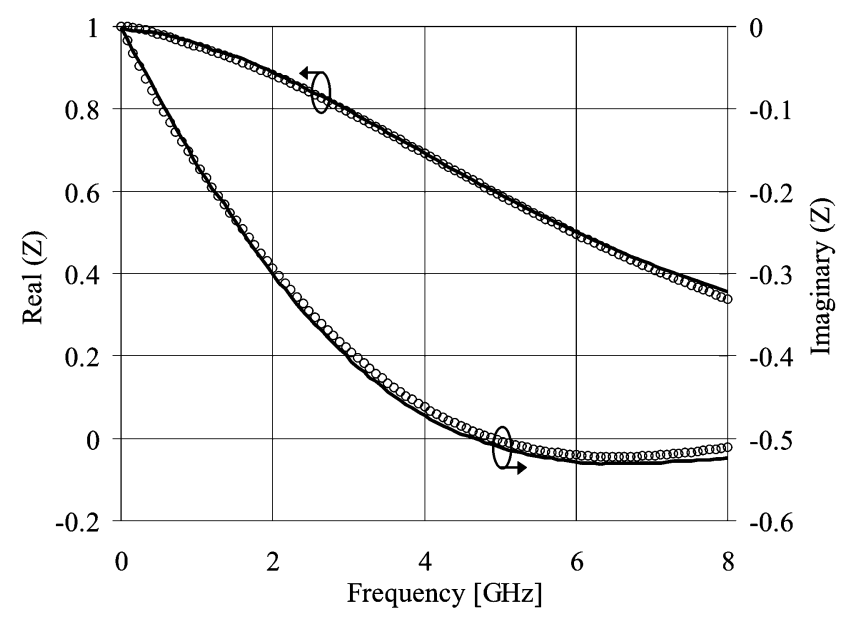

(b)

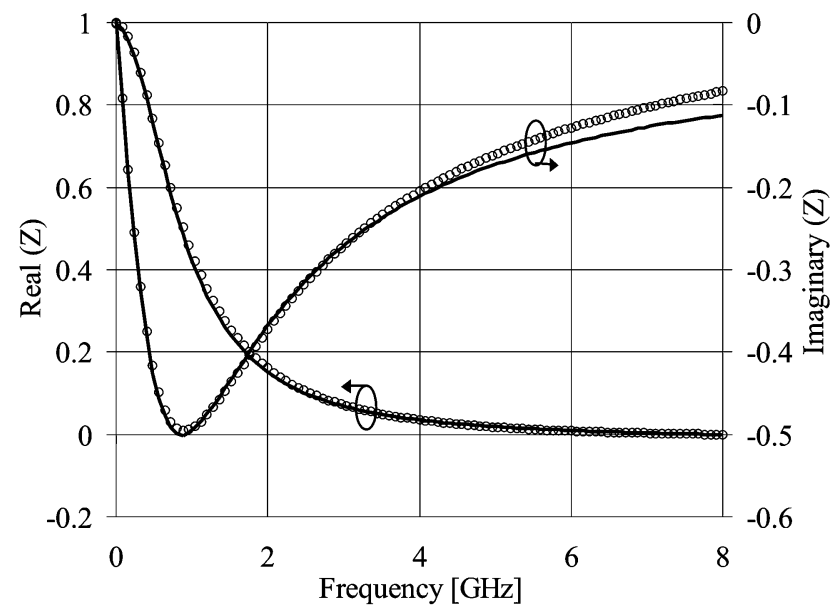

(d)

Fig. 13. The measured (continuous line) and simulated (points) S-parameters of the temperature compensated capacitor in ON- and OFF-state. (a), (b), (c), and (d) are the $\mathrm{S}_{11}$-parameters in the OFF-state, $\mathrm{S}_{21}$-parameters in the OFF-state, $\mathrm{S}_{11}$-parameters in the ON-state, and $\mathrm{S}_{21}$-parameters in the ON-state, respectively.

\& Schwarz ZVCE vector network analyzer and an HP 6634B dc voltage source. The capacitance value was calculated from the measured S-parameters. The transient capacitance was measured using a self-made, one-port vector network analyzer operating at $836 \mathrm{MHz}$. We noticed that the self-made one-port vector network analyzer had rather poor capacitance measurement accuracy. However, this is not a problem since it is meant to evaluate capacitance transient behavior rather than absolute values.

\section{Bridge Capacitor Temperature Dependence}

Fig. 9 shows a bridge-type capacitor used for temperature dependency measurements. The layer thicknesses are the same as in Table I, except that Metal 2 is $1-\mu \mathrm{m}$-thick gold. The length of the bridge is $372 \mu \mathrm{m}$, the width is $236 \mu \mathrm{m}$ in the middle of the bridge, and $316 \mu \mathrm{m}$ at the ends of the bridge.

Fig. 10(a) shows the capacitance as a function of voltage at $25^{\circ} \mathrm{C}$. Fig. 10(b) shows two cycles of the capacitance as a function of voltage at $55{ }^{\circ} \mathrm{C}$. At $55^{\circ} \mathrm{C}$ temperature change has caused the total in-plane stress in the bridge to become compressive. However, the bridge has not yet buckled and in the beginning of the first cycle, the capacitance is in its normal OFF state $(0.73 \mathrm{pF})$. The first control voltage cycle, the continuous line in Fig. 10(b), shows that the compressive stress has reduced the pull-in voltage and that the capacitance does not return to the OFF state when the control voltage is reduced to zero. The second control voltage cycle, the circles in Fig. 10(b), shows that the capacitance as a function of voltage is reproducible at that temperature, but the OFF state cannot be reached.

\section{Temperature-Compensated Capacitor}

Fig. 11 shows a photograph of the temperature compensated "Square" capacitor. The dimensions of the device are shown in Fig. 6 and in Table I. The capacitor has a frame surrounding the suspended electrode, to compensate for temperature change.

Fig. 12 shows the measured capacitance as a function of the voltage of the device at -30 and $+70^{\circ} \mathrm{C}$. Measurements show that the fluctuation of $C_{\mathrm{OFF}}$ is less than $6 \%, C_{\mathrm{ON}}$ is less than $2 \%$, and $U_{\text {pull-in }}$ is less than $5 \%$ over a $100{ }^{\circ} \mathrm{C}$ temperature range.

Fig. 13 shows the measured $\mathrm{S}_{11^{-}}$and $\mathrm{S}_{21}$-parameters of a temperature-compensated capacitor in both the OFF and the ON state. The continuous line in the figures is the measured data and the circles are the equivalent circuit data that is fitted to the measurement data. The equivalent circuit is shown in Fig. 7. Since the device is symmetric, only the $S_{11}$ - and $S_{21}$-parameters are shown. Table II shows the calculated equivalent circuit parameters. The measured ON capacitance is lower than designed. This 


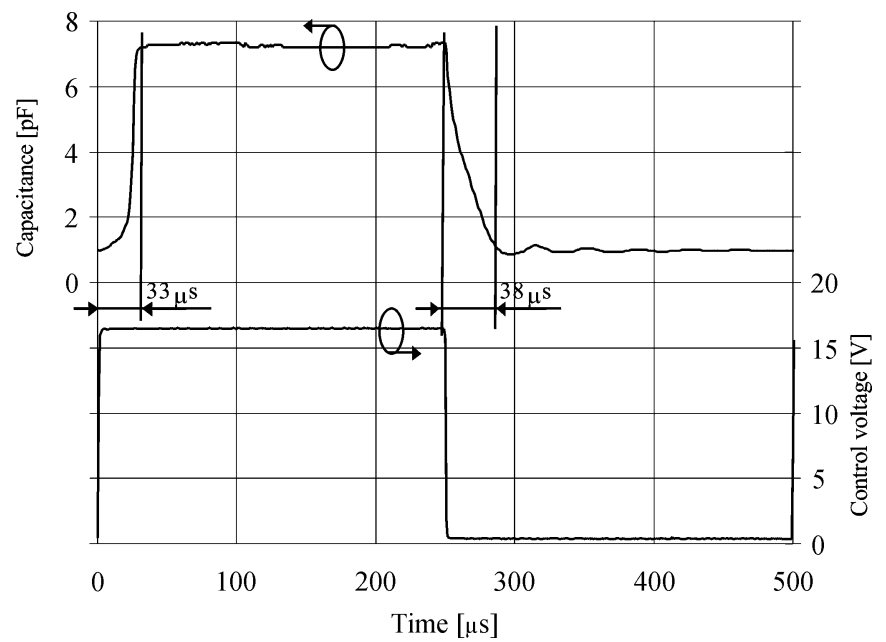

Fig. 14. Measured capacitance as a function of time.

TABLE III

SUMMARY OF MEASUREMENTS

\begin{tabular}{|c|c|c|}
\hline Temperature & $-30^{\circ} \mathrm{C}$ & $+70^{\circ} \mathrm{C}$ \\
\hline Measured devices & 38 & 38 \\
\hline Functional devices & 33 & 34 \\
\hline Designed $C_{\mathrm{OFF}}$ & $0.65 \mathrm{pF}$ & $0.65 \mathrm{pF}$ \\
\hline Average measured $C_{\mathrm{OFF}}$ & $0.95 \mathrm{pF}$ & $1.01 \mathrm{pF}$ \\
\hline Standard deviation & $0.04 \mathrm{pF}$ & $0.04 \mathrm{pF}$ \\
\hline Designed $C_{\mathrm{ON}}$ & $19 \mathrm{pF}$ & $19 \mathrm{pF}$ \\
\hline Average measured $C_{\mathrm{ON}}(10 \mathrm{~V})$ & $7.2 \mathrm{pF}$ & $7.2 \mathrm{pF}$ \\
\hline Standard deviation & $0.7 \mathrm{pF}$ & $0.7 \mathrm{pF}$ \\
\hline Designed $U_{\text {pull-in }}$ & $7.5 \mathrm{~V}$ & $7.5 \mathrm{~V}$ \\
\hline Average measured $\underline{U}_{\text {pull-in }}$ & $12.3 \mathrm{~V}$ & $12.8 \mathrm{~V}$ \\
\hline Standard deviation & $2.0 \mathrm{~V}$ & $2.1 \mathrm{~V}$ \\
\hline Average measured $U_{\text {Release }}$ & $5.3 \mathrm{~V}$ & $5.4 \mathrm{~V}$ \\
\hline Standard deviation & $1.0 \mathrm{~V}$ & $0.9 \mathrm{~V}$ \\
\hline
\end{tabular}

is due to the higher than estimated surface roughness between dielectric layer and the metal two layer. The surface roughness is about $60 \mathrm{~nm}$. However, it is visible from earlier fabrication runs that the target surface roughness about $15 \mathrm{~nm}$ can be achieved. The OFF capacitance of the device is higher than designed. In future, the design process should take into account suspended electrode warping that can increase the OFF capacitance.

Fig. 14 shows the MEM capacitor capacitance as a function of time measurement results. Measured $\tau_{\mathrm{ON}}$ and $\tau_{\mathrm{OFF}}$ are 33 and $38 \mu \mathrm{s}$, respectively.

A summary of 38 measured components from one wafer is presented in Table III. The table shows that one of the components was broken during the temperature cycling. Other damaged components broke during fabrication.
A device that has a $1-\mu$ m-thick suspended membrane was also fabricated. The surrounding temperature compensation frame that connects the membrane to the anchor points is $10 \mu \mathrm{m}$ thick and $30 \mu \mathrm{m}$ wide. With this process temperature-compensated bridge capacitors ranging in thicknesses of $0.3-1.5 \mu \mathrm{m}$ can be made. However, thin membranes of the fabricated devices buckle alot. This means that the in-plane intrinsic stress in the thick metal frame is more compressive than the intrinsic stress in the thin metal membrane.

\section{CONCLUSION}

This paper demonstrates a way to design temperaturecompensated MEM capacitors with a MEMS process that has a metal layer, dielectric layer, sacrificial layer, and second metal layer. Due to the simple process, the devices are very robust. The introduced temperature compensation does not in any way restrict design of the capacitance as a function of the voltage behavior. In addition, the temperature compensation method eliminates the effect of the in-plane intrinsic stress on the operation of the MEM capacitor. Measurements show that fabricated devices have good temperature stability. $C_{\mathrm{OFF}}$ changes less than $6 \%, C_{\mathrm{ON}}$ less than $2 \%$, and $U_{\text {pull-in }}$ less than $5 \%$, when the temperature is changed from -30 to $+70{ }^{\circ} \mathrm{C}$.

The presented temperature compensation technology can be expanded to many MEM devices that have suspended structure that requires isolation from the substrate stresses.

\section{ACKNOWLEDGMENT}

The RF MEM capacitors were fabricated by M. Trzmiel, C. Pisella, and S. Renard of Tronic's Microsystems, Grenoble, France. The authors are also indebted to P. Siukonen and M. Andersson of Nokia Research Center for their help in RF measurements. We would also like to thank J. Hyyryläinen of Nokia Research Center for the self-made, one-port vector network analyzer that was used for the capacitance transient measurements.

\section{REFERENCES}

[1] L. Dussopt and G. Rebeiz, "Intermodulation distortion and power handling in RF MEMS switches, varactors, and tunable filters," IEEE Trans. Microwave Theory Tech., vol. 51, pp. 1247-1256, 2003.

[2] D. Young and B. Boser, "A micromachined variable capacitor for monolithic low-noise VCO's," in IEEE Solid-State Sensors and Actuators Workshop Dig., June 1996, pp. 86-89.

[3] H. Nieminen, V. Ermolov, K. Nybergh, S. Silanto, and T. Ryhänen, "Microelectromechanical capacitors for RF applications," J. Micromech. Microeng., vol. 12, pp. 177-186, 2002.

[4] C. Goldsmith, A. Malczewski, Z. Yao, S. Chen, J. Ehmke, and D. Hinzel, "RF MEMS variable capacitors for tunable filters," Int. J. RF and Microwave CAE, vol. 9, pp. 362-374, 1999.

[5] D. Peroulis, P. Pacheco, K. Sarabandi, and L. Katehi, "Electromechanical considerations in developing low-voltage RF MEMS switches," IEEE Trans. Microwave Theory Tech., vol. 51, pp. 251-270, 2003.

[6] A. Margomenos and L. Katehi, "DC to $40 \mathrm{GHz}$ on-wafer package for RF MEMS switches," in Electrical Performance of Electronic Packaging, 2002, pp. 91-94.

[7] H. Tilmans, H. Ziad, H. Jansen, O. Di Monaco, A. Jourdain, W. De Raedt, X. Rottenberg, E. De Backer, A. Decaussernaeker, and K. Baert, "Wafer-level packaged RF-MEMS switches fabricated in a CMOS fab," in IEDM Tech. Dig., 2001, pp. 41.4.1-41.4.4.

[8] Y.-K. Park, Y.-K. Kim, H. Kim, D.-J. Lee, C.-J. Kim, B.-K. Ju, and J.-O. Park, "A novel thin chip scale packaging of the RF-MEMS devices using ultra thin silicon," in Conf. Proc. MEMS 03, 2003, pp. 618-621. 
[9] C. Goldsmith, J. Ehmke, A. Malczewski, B. Pillans, S. Eshelman, Z. Yao, J. Brank, and M. Eberly, "Lifetime characterization of capacitive RF MEMS switches," in IEEE MTT-S Int. Microwave Symp. Dig., vol. 1, 2001, pp. 227-230.

[10] E. Fourn, A. Pothier, C. Champeaux, P. Tristant, A. Catherinot, P. Blondy, G. Tanne, E. Rius, C. Person, and F. Huret, "MEMS switchable interdigital coplanar filter," IEEE Trans. Microwave Theory Tech., vol. 51, pp. 320-324, 2003.

[11] K. Hong-Teuk, P. Jae-Hyoung, K. Yong-Kweon, and K. Youngwoo, "Millimeter-wave micromachined tunable filters," in IEEE MTT-S Int. Microwave Symp. Dig., vol. 3, 1999, pp. 1235-1238.

[12] F. Plötz, S. Michaelis, R. Aigner, H.-J. Timme, J. Binder, and R. Noe, "A low-voltage torsional actuator for application in RF-microswitches," Sens. Actuators A, vol. 92, pp. 312-317, 2001.

[13] A. Dec and K. Suyama, "Microwave MEMS-based voltage-controlled oscillators," IEEE Trans. Microwave Theory Tech., vol. 48, pp. 1943-1949, 2000.

[14] H. Nieminen, T. Ryhänen, V. Ermolov, and S. Silanto, "Method and Structure That Forms Firm Boundary Conditions for Metal Suspended Structures," U. S. Patent 6557 413, May 6, 2003.

[15] G. Rebeiz, RF MEMS Theory, Design, and Technology, NJ: Wiley, 2003, pp. $21-57$.

[16] T. Veijola, T. Tinttunen, H. Nieminen, V. Ermolov, and T. Ryhänen, "Gas damping model for a RF MEMS switch and its dynamic characteristics," in IEEE MTT-S Int. Microwave Symp. Dig., vol. 2, 2002, pp. 1213-1216.

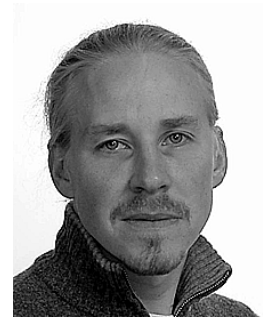

Heikki Nieminen received the M.S. (Tech.) degree in engineering physics from the Helsinki University of Technology (HUT), Espoo, Finland, in 1999.

$\mathrm{He}$ is currently working toward the Doctor of Science (Tech.) degree at HUT. He is currently a Research Engineer with the Nokia Research Center, Helsinki, Finland. His research interests are focused on design of microelectromechanical devices, reconfigurable radio frequency circuits, and sensor networks. He holds one patent.

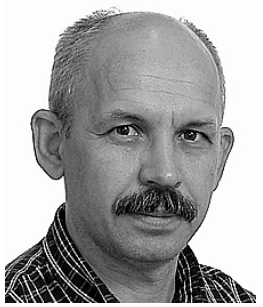

Vladimir Ermolov received the M.Sc. degree in engineering physics and the Ph.D. degree in engineering physics from the Moscow Engineering Physics University (MEPhI), Moscow, Russia, in 1981 and 1986, respectively.

From 1981 to 1998, he was Senior Research Assosiate with the Laboratory of Dielectric Devices, MEPhI, during which time he was a Visiting Researcher numerous times with the Department of Physics, Helsinki University, Helsinki, Finland, and Fraunhofer Institute of Nondestructive testing, Saarbrücken, Germany. In 1998, he joined Nokia Research Center, Helsinki, as Senior Research Engineer, where he has been a Project Manager for several MEMS projects. During his professional career, he has been involved in areas of sensors for measurements of ocean parameters, acoustic devices for signal processing, surface acoustic wave (SAW) devices, variable acoustic devices, acoustic effects in magnetic materials and their technical applications, nonlinear effects in solid state, acoustical and nondestructive evaluation, and MEMS. He has authored and coauthored 47 scientific publications. He holds and co-holds 22 patents.

Samuli Silanto, photograph and biography not available at the time of publication.

Kjell Nybergh, photograph and biography not available at the time of publication.

Tapani Ryhänen was born in Helsinki, Finland, on July 7, 1959. He received the M.S. (Tech.) degree in engineering physics and the Doctor of Science (Tech.) degree in applied electronics from the Helsinki University of Technology (HUT) in 1986 and 1992, respectively.

From 1992 to 1995, he was with Vaisala Technologies Inc., where he designed micromechanical pressure and angular rate sensors. In February 1995, he joined Nokia Telecommunications, where he was a Research and Development Manager and a Senior Product Manager. In December 1998, he joined the Nokia Research Center, Helsinki, as a Principal Scientist, where he was responsible for the MEMS research program. Since April 2000, he has headed the Microsystem Technologies Group as a Senior Research Manager. He has authored and coauthored several publications on the theory, design, and characterization of ultralow noise superconducting thin-film magnetometers and microelectromechanical sensors, actuators, and systems. He holds six patents with several pending, which are related to micromechanical devices and systems. His current research focus is in the fields of RF MEMS, sensors, and integration of microsystems into portable electronics. 